\title{
Negative-pressure dressing management of recurrent postoperative epidural hematoma and uncontrollable intraoperative hemorrhage in posterior spine surgery: report of 4 cases
}

\author{
Kenneth C. Foxx, MD, Addisu Mesfin, MD, and Robert Molinari, MD \\ Departments of Orthopaedic Surgery and Neurosurgery, University of Rochester Medical Center, Rochester, New York
}

There is a paucity of literature describing the management of recurrent symptomatic postoperative epidural hematoma or uncontrollable intraoperative hemorrhage in posterior spine surgery. Traditional management with hematoma evacuation and wound closure over suction drains may not be effective in certain cases, and it can lead to recurrence and neurological injury. The authors report 3 cases of recurrent symptomatic postoperative epidural hematoma successfully managed with novel open-wound negative-pressure dressing therapy (NPDT), as well as 1 case of uncontrollable intraoperative hemorrhage that was primarily managed with the same technique.

The 3 patients who developed a postoperative epidural hematoma became symptomatic 2-17 days after the initial operation. All 3 patients underwent at least 1 hematoma evacuation and wound closure over suction drains prior to recurrence with severe neurological deficit and definitive management with NPDT. One patient was managed primarily with NPDT for uncontrollable intraoperative hemorrhage during posterior cervical laminectomy.

All 4 patients had significant risk factors for postoperative epidural hematoma. NPDT for 3-9 days with delayed wound closure was successful in all patients. The 3 patients with recurrent symptomatic postoperative epidural hematoma had significant improvement in their severe neurological deficit. None of the patients developed a postoperative wound infection, and none of the patients required transfusion due to NPDT.

NPDT with delayed wound closure may be an option in certain patients, when wound closure over suction drains is unlikely to prevent further neurological injury.

https://thejns.org/doi/abs/10.3171/2017.5.SPINE17387

KEY WORDS negative-pressure dressing therapy; uncontrollable intraoperative hemorrhage; recurrent symptomatic postoperative epidural hematoma

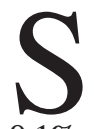
YMPTOMATIC postoperative epidural hematoma is a rare, although potentially devastating, complication of spine surgery, with an overall incidence of $0.1 \%-0.41 \% .^{1,3,4,12}$ The incidence may be higher in patients undergoing posterior cervical $(1.5 \%)^{10}$ or thoracic $(4.46 \%)^{3}$ surgery. Asymptomatic epidural hematoma after spine surgery is quite common. In one series, $58 \%$ of patients undergoing lumbar decompression had postoperative MRI evidence of epidural hematoma compressing the thecal sac beyond its preoperative state, but none of these patients were symptomatic. ${ }^{17}$

Postoperative epidural hematomas that are symptom- atic and require evacuation usually present soon after the initial operation..$^{15}$ The median time to symptom onset was 2.7 hours after surgery in 1 series. ${ }^{1}$ However, there are multiple reports of delayed symptomatic postoperative epidural hematoma formation..$^{16,18}$ These patients usually first develop severe pain at the operative site, followed by radicular pain, paresthesias, and neurological deficit. ${ }^{14,15,18}$ The rapid identification of symptoms due to a postoperative epidural hematoma and emergent surgical evacuation increases the likelihood of neurological recovery., ${ }^{1,12}$

Preoperative risk factors for the development of an epidural hematoma after spine surgery include coagulopathy,

ABBREVIATIONS HCT = hematocrit; INR = international normalized ratio; IV = intravenous; NPD = negative-pressure dressing; NPDT = NPD therapy; NSAID = nonsteroidal antiinflammatory drug; OR = operating room.

SUBMITTED March 29, 2017. ACCEPTED May 30, 2017.

INCLUDE WHEN CITING Published online December 1, 2017; DOI: 10.3171/2017.5.SPINE17387. 
previous spinal surgery, age over 60 years, excessive alcohol consumption, nonsteroidal antiinflammatory drug (NSAID) use, and Rh-positive blood type. ${ }^{1,4,11}$ Operative and postoperative risk factors include multilevel procedure, hemoglobin level less than $10 \mathrm{~g} / \mathrm{dl}$, intraoperative blood loss greater than $1 \mathrm{~L}$, and international normalized ratio (INR) greater than 2.0 within 48 hours of surgery.,11 Postoperative use of NSAIDs following posterior cervical surgery may also increase the risk of epidural hematoma. ${ }^{10}$

Recurrent symptomatic postoperative epidural hematoma following surgical evacuation is extremely rare, and there are only a few cases reported in the literature. ${ }^{2,9}$ Given the paucity of literature, there is little guidance for managing these patients. There is also limited direction for the management of uncontrollable intraoperative hemorrhage in posterior spine surgery. We report 3 cases of recurrent postoperative epidural hematoma and 1 case of uncontrollable intraoperative hemorrhage managed with open-wound negative-pressure dressing therapy (NPDT).

\section{Case Reports}

At a single institution, 2 surgeons employed a novel open-wound NPDT technique in 4 patients between September 2016 and January 2017. We retrospectively reviewed the medical records of these 4 patients. Three of the patients were male and 1 was female. The patients were in their 70s or 80s, had medical comorbidities, and had significant risk factors for postoperative epidural hematoma formation.

Three of the patients had a recurrent postoperative epidural hematoma causing severe neurological deficit and had previously undergone at least 1 hematoma evacuation and closure over suction drains. Two of these patients initially underwent cervical posterolateral arthrodesis and laminectomy. One patient underwent lumbar laminectomy that was extended to evacuate hematoma tracking in the epidural space during the first return to the operating room (OR). The decision to employ NPDT was made by the attending surgeon based on the assessment that repeat hematoma evacuation and closure over suction drains was unlikely to prevent further neurological injury.

One patient was primarily managed with NPDT for uncontrollable intraoperative hemorrhage while undergoing cervical laminectomy. In this unique situation, we were operating on a patient with pharmacologically elevated arterial pressures necessitated by an acute stroke. The attending surgeon judged the diffuse hemorrhage from the posterior cervical musculature and epidural space to pose an unacceptably high probability of postoperative epidural hematoma formation and neurological injury. Based on this assessment, the decision to employ NPDT was made.

\section{Negative-Pressure Dressing Application Technique}

The same technique was used in all 4 patients for application of the negative-pressure dressing (NPD). All procedures were performed in the OR under general anesthesia. Two of the 3 patients who had a recurrent postoperative epidural hematoma underwent evacuation in the OR prior to NPD placement. One patient had already undergone hematoma evacuation at the bedside and had packing in

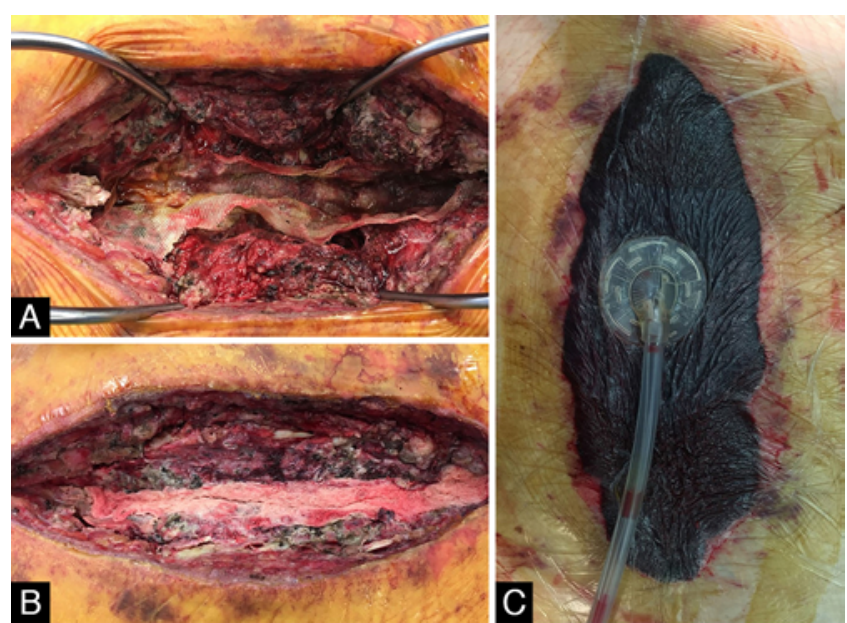

FIG. 1. Intraoperative images showing application of the open-wound NPD. A: Adaptic nonadherent dressing was applied over the dura.

B: The wound was packed with moist gauze dressing. C: A VAC dressing was applied to the wound. Figure is available in color online only.

place, which was removed in the OR. The patient who was primarily managed with NPDT for uncontrollable intraoperative hemorrhage was already under general anesthesia and had undergone cervical laminectomy.

Adaptic nonadherent dressing (Johnson \& Johnson) was placed on the dura in all cases, and the wounds were packed with moist gauze. A VAC (Kinetic Concepts, Inc.) dressing was placed and connected to $-50 \mathrm{~mm} \mathrm{Hg}$ of intermittent suction (Fig. 1). The intermittent suction setting involved the cyclical application of suction for 5 minutes and release for 2 minutes.

In 1 patient, the edges of the posterior cervical wound were approximated with 2-0 nylon retention sutures after application of the sponge component of the VAC dressing. The flange component of the dressing containing the suction tubing was then applied (Fig. 2). This was necessary because the edges of the cervical wound had spread quite widely and would have made it difficult to maintain negative-pressure suction.

\section{Case 1}

This 72-year-old woman with a history of diabetes, hyperlipidemia, hypertension, transient ischemic attack, coronary artery disease, and coronary stent placement 6 months prior, on aspirin $81 \mathrm{mg}$ and clopidogrel $75 \mathrm{mg}$ daily, underwent L2-4 bilateral laminectomies for lumbar stenosis. Clopidogrel and aspirin were resumed on postoperative Day 1 given the patient's significant cardiac history and her cardiologist's recommendations. On postoperative Day 2, she developed severe low-back and bilateral lowerextremity pain associated with mild right extensor hallucis longus and dorsiflexion weakness. MRI demonstrated a large surgical-site hematoma with significant compression of the cauda equina (Fig. 3).

The patient underwent emergent evacuation of the surgical-site hematoma, as well as T12-L1 and L4-5 bilateral laminectomies to evacuate hematoma tracking in the epidural space and wound closure over suction drains. 


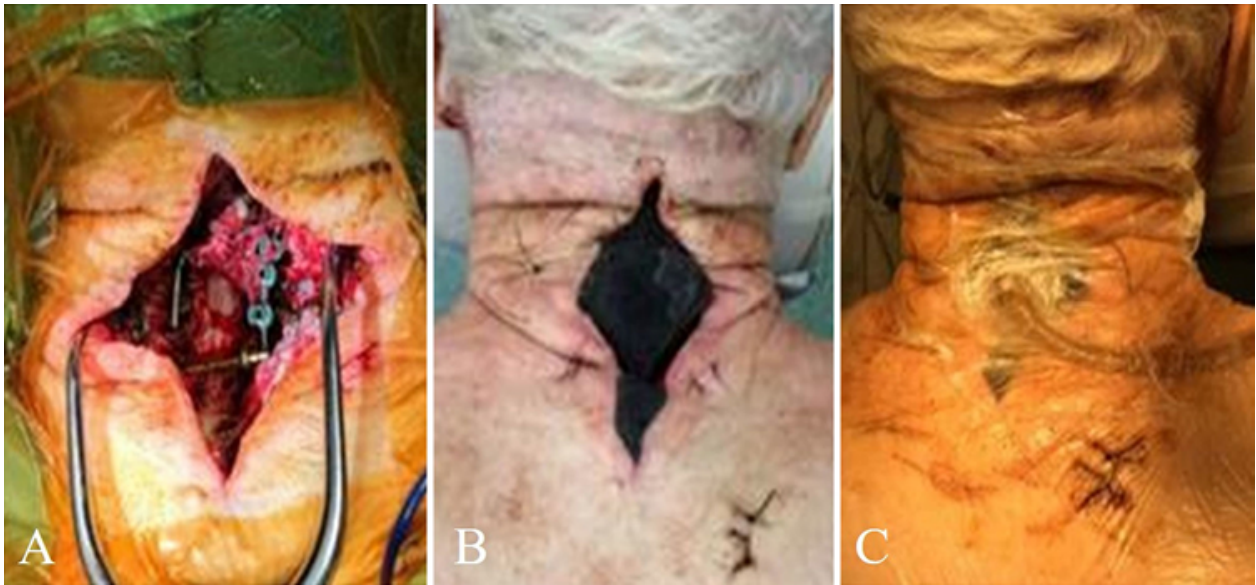

FIG. 2. Intraoperative images showing removal of the open-wound NPD. A: After removal of the NPD, no significant source of hemorrhage was seen in the surgical wound. B: After removal of the suction flange and adhesive dressing, the sponge component of the VAC dressing is seen with overlying retention sutures. C: The NPD was inspected and noted to be clean and functioning prior to removal. Figure is available in color online only.

Despite initially moving all extremities after surgery, the patient developed paraplegia, loss of rectal tone, and saddle anesthesia an hour after surgery. She was then taken back to the OR, and the recurrent hematoma was evacuated. The wound was again closed over multiple suction drains. She did not recover neurological function after the second hematoma evacuation.

Another MRI study was obtained, which showed a compressive epidural hematoma. The patient was taken back to the OR for evacuation of the hematoma and placement of an NPD. Her hematocrit (HCT) ranged from $26 \%$ to $30 \%$ during the period of NPDT, and she did not require transfusion. Prophylactic intravenous (IV) cefazolin (1000 mg every 8 hours) was given for the duration of NPDT. Three days later, the patient was taken back to the OR for removal of the NPD, washout, and closure. She was recently seen at her 7-month follow-up appointment. She is now neurologically intact, ambulating without assistance, and living at home, but continues to self-catheterize for neurogenic urinary retention.

\section{Case 2}

This 72-year-old woman with a history of Stage IV squamous cell carcinoma of the tongue, Parkinson's disease, diabetes, and cirrhosis presented to the emergency department with rapidly progressive tetraparesis. MRI of the cervical spine demonstrated diffuse cervical spondylosis, multilevel severe canal stenosis, and a contrastenhancing lesion involving the posterior elements of C-3 and C-4. She underwent C2-T1 posterolateral fusion and C3-7 bilateral laminectomies.

On postoperative Day 8, she developed acutely worsening neck pain and progressive weakness and sensory deficit in the bilateral upper extremities. She was not on anticoagulant or antiplatelet medications, but she did have a baseline coagulopathy secondary to cirrhosis, with an INR of 1.6. She was emergently taken to the OR, where a surgical-site hematoma under significant pressure was evacuated and the wound was closed over multiple suction drains. After an initial period of improvement, she devel- oped acutely worsening neck pain and tetraplegia 2 days later. Her wound was opened at the bedside, a hematoma under significant pressure was evacuated, and the wound was packed. She was then taken to the OR for NPD placement.

The patient's neurological function gradually improved over the next several days. Her HCT ranged from $22 \%$ to $26 \%$ during the period of NPDT, and she did not require transfusion. Her INR remained elevated due to her under-
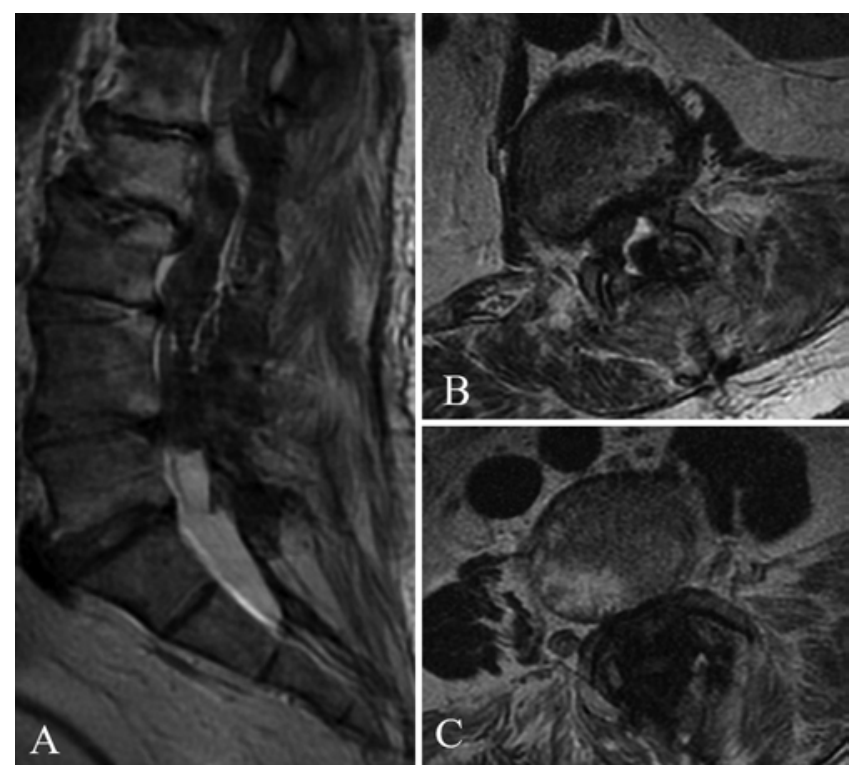

FIG. 3. Case 1. T2-weighted MR images of the patient's lumbar spine obtained on postoperative Day 2. A: Midsagittal image demonstrating significant compression of the cauda equina at L2-4 where laminectomies have been performed. There is also compressive hematoma tracking in the epidural space, causing significant compression at L-1 and L-5. B: Axial image obtained at the L1-2 level demonstrating hematoma tracking in the epidural space, causing significant compression just below the conus medullaris. C: Axial image obtained at the L4-5 level showing the epidural hematoma causing significant compression of the cauda equina. 


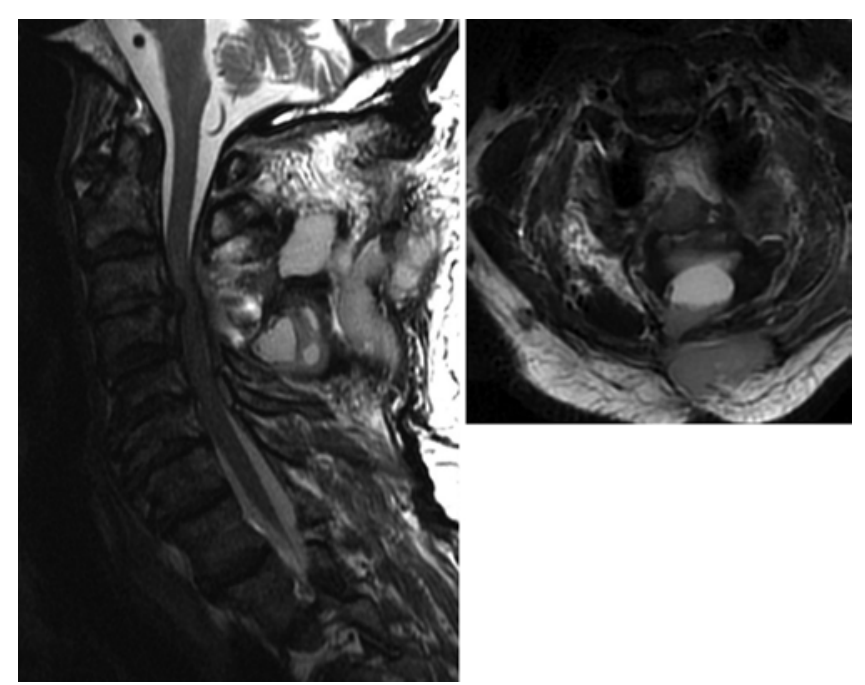

FIG. 4. Case 3. Midsagittal (left) and axial (right) T2-weighted MR images of the patient's cervical spine obtained on postoperative Day 17. The axial image is at the C3-4 level. There was a large epidural hematoma causing significant compression of the cervical spinal cord.

lying coagulopathy and ranged from 1.5 to 1.9 . Prophylactic IV cefazolin (1000 mg every 8 hours) was given for the duration of NPDT. The patient was taken back to the OR 9 days later for removal of the NPD, wound washout, and closure. She was eventually discharged to hospice care and has since died due to her underlying disease. At the time of discharge, she had recovered at least antigravity strength or better in all muscle groups.

\section{Case 3}

This 81-year-old man with a history of diabetes and hypertension presented to the emergency department with rapidly progressive myelopathy after falling from standing several weeks prior. MRI of the cervical spine demonstrated severe canal stenosis C3-4 causing cord compression and intramedullary signal change. He underwent C3-4 posterolateral fusion and bilateral laminectomies.

The patient was started on therapeutic anticoagulation on postoperative Day 1 after being diagnosed with a pulmonary embolism and deep venous thrombosis. Seventeen days after posterior cervical decompression and fusion, the patient acutely developed severe neck pain and tetraplegia. He was on warfarin with an INR of 2.3 at that time. MRI demonstrated a surgical-site hematoma with significant cord compression (Fig. 4). Anticoagulation was reversed with a prothrombin complex concentrate agent and IV vitamin $\mathrm{K}$. The patient was emergently taken to the OR, a large hematoma under significant pressure was evacuated, and the wound was closed over a suction drain. The patient did not recover neurological function after surgery, and MRI demonstrated a recurrent epidural hematoma (Fig. 5).

The patient was taken back to the OR, the hematoma was evacuated, and an NPD was placed. The patient gradually recovered neurological function over the next several days. The patient's HCT ranged from $26 \%$ to $28 \%$ during the period of NPDT, and he did not require transfusion.
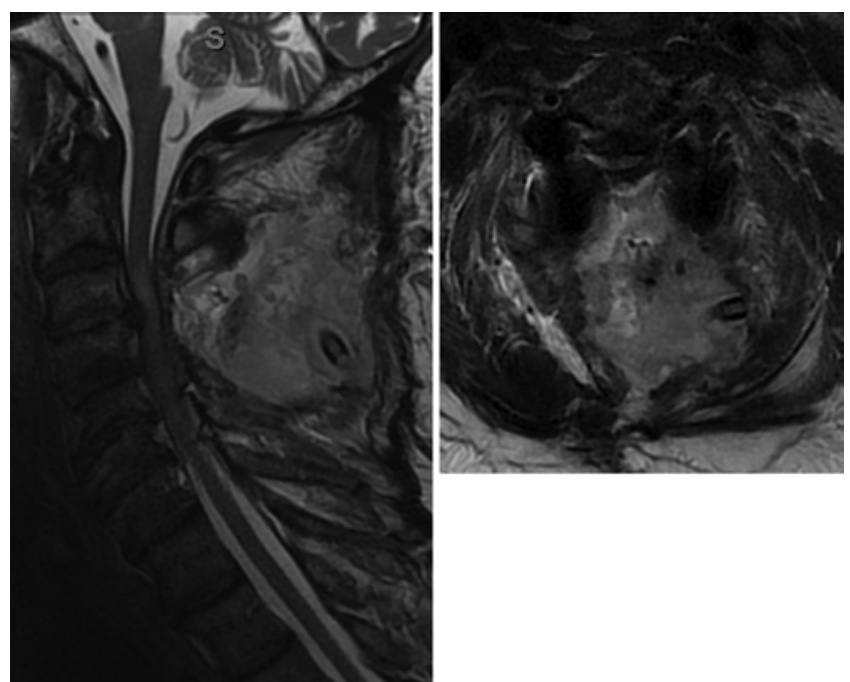

FIG. 5. Case 3. Midsagittal (left) and axial (right) T2-weighted MR images of the patient's cervical spine obtained after the first hematoma evacuation and closure over a suction drain. The axial image is at the C3-4 level. There was a recurrent epidural hematoma causing compression of the cervical spinal cord associated with intramedullary signal change.

Prophylactic IV cefazolin (2000 mg every 8 hours) was given for the duration of NPDT. On postoperative Day 9, the patient was taken back to the OR, the NPD was removed, and the wound was debrided and closed. The patient was recently seen at his 3 -month follow-up visit. His neurological condition has improved compared with his initial presentation. He is now living at home, ambulating with a walker, and voiding normally.

\section{Case 4}

This 84-year-old man with a history of hypertension presented to the emergency department with neck pain after falling from standing. CT and subsequent MRI of the cervical spine showed disruption of the $\mathrm{C} 4-5$ disc space with anterolisthesis of C-4 on C-5 and a perched left C4-5 facet. The patient underwent manual reduction of the perched left facet C4-5 and C3-T1 posterolateral fusion without decompression.

Neurological examination after surgery revealed a new left homonomous hemianopsia and significant right upperand lower-extremity weakness. MRI of the brain demonstrated scattered areas of diffusion restriction involving multiple vascular territories of the right hemisphere, which explained the patient's visual deficit but not his weakness. MRI of the cervical spine showed buckling of the ligamentum flavum at $\mathrm{C} 4-5$ and compression of the right spinal cord. The neurology service was consulted and supported the plan for emergent return to the OR if mean arterial pressure was maintained above $90 \mathrm{~mm} \mathrm{Hg}$.

The patient was taken back to the OR and underwent C4-5 bilateral laminectomies. There was uncontrollable intraoperative epidural and muscular bleeding, and the decision was made to primarily place an NPD. The total intraoperative blood loss was $500 \mathrm{ml}$; postoperatively, the patient's HCT was $24 \%$, his platelet count was $95 \times 10^{3} / \mu 1$, 
and his INR was 1.2. He did not receive any transfusion of blood products.

During the period of NPDT, the patient's HCT ranged from $24 \%$ to $28 \%$, and he did not require transfusion. Prophylactic IV cefazolin (1000 mg every 8 hours) was given for the duration of NPDT. Seven days after the initial operations, the patient was taken back to the OR, the NPD was removed, and the wound was debrided and closed. His neurological examination findings remained stable compared with after the initial operation, but he developed several medical complications over the following weeks, was transferred to hospice care, and subsequently died.

\section{Discussion}

Recurrent postoperative epidural hematoma following surgical evacuation is extremely rare, and there are only a few case reports in the literature. ${ }^{2,9}$ Recurrent hematoma after surgical evacuation or conservative management of a spontaneous spinal epidural hematoma is also quite rare. Luo et al. ${ }^{13}$ reported that only 11 such cases existed in the English literature.

Surgeons have little direction for managing patients with recurrent symptomatic postoperative epidural hematoma. Our 3 cases of recurrent surgical-site hematoma successfully managed with NPDT provide some guidance. All 3 patients were elderly with medical comorbidities and coagulopathy. The first patient was coagulopathic due to dual antiplatelet therapy, the second due to cirrhosis, and the third was on therapeutic anticoagulation. Our patients developed delayed symptomatic postoperative epidural hematomas 2, 8, and 17 days after surgery. All 3 patients underwent at least 1 hematoma evacuation and closure over suction drains prior to NPD placement. In each case, another epidural hematoma evacuation and closure over suction drains was determined to be unlikely to prevent further neurological injury by the attending surgeon.

Coagulopathy is a significant and obvious risk factor for the development of postoperative epidural hematoma., ${ }^{4,11}$ Therapeutic anticoagulation after spine surgery is known to be associated with serious complications. ${ }^{5,7}$ However, this risk must be weighed against the risk of forgoing treatment of the underlying condition. In patients with recently placed coronary stents, the proper timing for the resumption of antiplatelet medications after semielective spine surgery is unclear. ${ }^{8}$ In practice, operating on patients with considerable risk factors for postoperative epidural hematoma can sometimes be unavoidable.

Our fourth case was primarily managed with NPDT because of intraoperative hemorrhage that could not be controlled. This was presumably caused by pharmacologically elevated mean arterial pressures, needed for cerebral perfusion in that patient. The attending surgeon determined that the ongoing and uncontrollable epidural and muscular bleeding posed an unacceptably high risk of neurological injury from a compressive surgical-site hematoma. It is difficult to quantify specific criteria for primary employment of NPDT, particularly given its use in only 1 patient in this series. There was rapid blood loss totaling $500 \mathrm{ml}$ for the procedure, but the patient did not receive any transfusion and the postoperative HCT was $24 \%$. The decision to use NPDT primarily was based on the subjective assess- ment that there was significant diffuse hemorrhage in the surgical wound from innumerable sources that could not be identified or controlled.

The same technique was used to apply the NPD in all 4 cases. The NPD suction device was set to $-50 \mathrm{~mm} \mathrm{Hg}$ of intermittent suction with cyclical application of suction for 5 minutes and release for 2 minutes. The effective range of therapeutic pressure is -40 to $-150 \mathrm{~mm} \mathrm{Hg}$ in settings where negative-pressure wound therapy is commonly used. ${ }^{6}$ A typical intermittent setting is 5 minutes of suction cycled with 2 minutes of release. ${ }^{6}$ We made the decision to use minimal negative-pressure settings and to increase those settings if necessary. Higher pressure settings would presumably also lead to increased blood loss. If there had been inadequate drainage from the wound or if the system was not holding suction, we likely would have changed the suction to continuous and then slowly increased the pressure setting.

All patients were treated with skin flora antibiotic prophylaxis (cefazolin) while the NPD was in place. Therapy was maintained for 3-9 days prior to removal, wound washout, and closure. None of our patients developed a wound infection after definitive closure, and no patient required a transfusion during the period of NPDT.

Luo et al. ${ }^{13}$ successfully managed a patient who developed a symptomatic postoperative epidural hematoma, following laminectomy and evacuation of a spontaneous epidural hematoma, using NPDT. The authors reported removing a few stitches at the bottom of the incision, evacuating the hematoma, and inserting the foam component of the dressing into the wound. Our technique differs in several ways from that described by Luo et al. Opening and packing the entire wound prior to placement of the NPD would seem to decrease the chance of compressive hematoma reaccumulation and prevent mass effect on underlying neural elements from dressing components. This allows for safe expansion of the technique to cases involving the thoracic and cervical spine. Wound packing prior to placement of the NPD and setting the pressure to $-50 \mathrm{~mm}$ $\mathrm{Hg}$ intermittent suction (compared with $-125 \mathrm{~mm} \mathrm{Hg}$ continuous suction described by Luo et al.) allowed for sufficient evacuation of hematological products in our patients, while also providing a tamponade effect. Indeed, none of our patients had significant decreases in HCT or required transfusion during the period of NPDT.

Although we successfully managed 4 patients with NPDT, our series is admittedly small, and there are several theoretical disadvantages to our technique. There is likely a considerable risk of wound infection, which we tried to mitigate with antibiotic prophylaxis. Devitalization of the wound edges from prolonged open-wound NPDT could lead to wound healing issues. We tried to reduce this risk with washout and debridement of the wounds after removal of the NPD and before definitive closure. There is a risk of continued blood loss with NPDT, which could necessitate transfusion. Although not seen in our series, a recognized or unrecognized cerebrospinal fluid leak in the setting of an open wound with pressurized drainage could lead to meningitis or complications associated with intracranial hypotension.

Despite these risks, open-wound NPDT with delayed wound closure is an option in certain patients in whom 
closure over suction drains is deemed unlikely to prevent further neurological deterioration. Larger series with the technique implemented in a diverse patient population and wider range of clinic scenarios are needed. Nevertheless, our report provides some direction in an area where there is little guidance.

\section{Conclusions}

Recurrent symptomatic postoperative epidural hematoma and uncontrollable intraoperative hemorrhage can be difficult to manage, and there are a very limited number of publications that address this topic. Wound closure over suction drains may not be effective in some cases and can lead to neurological injury. Our novel open-wound NPDT technique with delayed wound closure is a viable alternative in certain patients and may allow time for correction of coagulopathy while preventing further neurological deterioration.

\section{References}

1. Amiri AR, Fouyas IP, Cro S, Casey AT: Postoperative spinal epidural hematoma (SEH): incidence, risk factors, onset, and management. Spine J 13:134-140, 2013

2. Anno M, Yamazaki T, Hara N, Hayakawa K: Recurrent postoperative spinal epidural hematoma in a patient with protein S deficiency. Case Rep Orthop 2015:536592, 2015

3. Aono H, Ohwada T, Hosono N, Tobimatsu H, Ariga K, Fuji T, et al: Incidence of postoperative symptomatic epidural hematoma in spinal decompression surgery. J Neurosurg Spine 15:202-205, 2011

4. Awad JN, Kebaish KM, Donigan J, Cohen DB, Kostuik JP: Analysis of the risk factors for the development of postoperative spinal epidural haematoma. J Bone Joint Surg Br 87:1248-1252, 2005

5. Barnes B, Alexander JT, Branch CL Jr: Postoperative Level 1 anticoagulation therapy and spinal surgery: practical guidelines for management. Neurosurg Focus 17(4):E5, 2004

6. Birke-Sorensen H, Malmsjo M, Rome P, Hudson D, Krug E, Berg L, et al: Evidence-based recommendations for negative pressure wound therapy: treatment variables (pressure levels, wound filler and contact layer) - steps towards an international consensus. J Plast Reconstr Aesthet Surg 64 Suppl:S1S16, 2011

7. Cain JE Jr, Major MR, Lauerman WC, West JL, Wood KB, Fueredi GA: The morbidity of heparin therapy after development of pulmonary embolus in patients undergoing thoracolumbar or lumbar spinal fusion. Spine (Phila Pa 1976) 20:1600-1603, 1995

8. Carragee EJ, Golish SR, Scuderi GJ: A case of late epidural hematoma in a patient on clopidogrel therapy postoperatively: when is it safe to resume antiplatelet agents? Spine J 11:e1e4, 2011
9. Caruso R, Pesce A, Wierzbicki V, Marrocco L: Recurrent spinal epidural hematoma: case report. Neurochirurgie 59:105-107, 2013

10. Goldstein CL, Bains I, Hurlbert RJ: Symptomatic spinal epidural hematoma after posterior cervical surgery: incidence and risk factors. Spine J 15:1179-1187, 2015

11. Kou J, Fischgrund J, Biddinger A, Herkowitz H: Risk factors for spinal epidural hematoma after spinal surgery. Spine (Phila Pa 1976) 27:1670-1673, 2002

12. Lawton MT, Porter RW, Heiserman JE, Jacobowitz R, Sonntag VK, Dickman CA: Surgical management of spinal epidural hematoma: relationship between surgical timing and neurological outcome. J Neurosurg 83:1-7, 1995

13. Luo XB, Zhou X, Wang Q, Cai XJ, Luo ZP, Ma YZ: The classification of recurrent spinal epidural hematoma: a review of the literature and a comparison with the cases. Eur Spine J 25 (Suppl 1):224-229, 2016

14. Morse K, Weight M, Molinari R: Extensive postoperative epidural hematoma after full anticoagulation: case report and review of the literature. J Spinal Cord Med 30:282-287, 2007

15. Scavarda D, Peruzzi P, Bazin A, Scherpereel B, Gomis P, Graftieaux JP, et al: [Postoperative spinal extradural hematomas. 14 cases.] Neurochirurgie 43:220-227, 1997 (Fr)

16. Sokolowski MJ, Dolan M, Aminian A, Haak MH, Schafer MF: Delayed epidural hematoma after spinal surgery: a report of 4 cases. J Spinal Disord Tech 19:603-606, 2006

17. Sokolowski MJ, Garvey TA, Perl J II, Sokolowski MS, Cho W, Mehbod AA, et al: Prospective study of postoperative lumbar epidural hematoma: incidence and risk factors. Spine (Phila Pa 1976) 33:108-113, 2008

18. Uribe J, Moza K, Jimenez O, Green B, Levi AD: Delayed postoperative spinal epidural hematomas. Spine J 3:125-129, 2003

\section{Disclosures}

Dr. Mesfin reports receipt of a research fellowship grant from Globus Medical and research grants from J. Robert Gladden Society.

\section{Author Contributions}

Conception and design: all authors. Acquisition of data: all authors. Analysis and interpretation of data: all authors. Drafting the article: all authors. Critically revising the article: all authors. Reviewed submitted version of manuscript: all authors. Approved the final version of the manuscript on behalf of all authors: Molinari. Administrative/technical/material support: all authors. Study supervision: all authors.

\section{Correspondence}

Robert Molinari, University of Rochester Medical Center, Orthopaedics and Rehabilitation, 601 Elmwood Ave., Box 665, Rochester, NY 14642.email: robert_molinari@urmc.rochester.edu. 